\title{
True identity of a diffraction pattern attributed to valyl tRNA
}

SIR - We have examined in detail several publications by H.H. Paradies. One is a report in Nature on 11 April 1970 about single crystals of a valine-specific tRNA from yeast '. We find that the diffraction pattern attributed to valyl tRNA in Fig. 2 of that article is in fact an X-ray photograph from a crystal of human carbonic anhydrase B. The circumstances are such that we see no alternative but to conclude that this was a deliberate misrepresentation.

Even a rather superficial reading of the paper raises suspicions.

First, there are implausibilities. The crystal that produces Figs 1 and 2 diffracts exceptionally well for a macromolecular crystal of the small size reported even in comparison with the best tRNA crystals yet characterized. That such crystals should suddenly be "completely destroyed" after short X-ray exposure is not plausible. It is also unbelievable that Fig. 3 does not show a beam-stop shadow.

Second, there are internal inconsistencies. Contrary to the statement that "proper alignment . . . could not be achieved", Figs 1 and 2 are aligned to within $1^{\circ}$ and $0.3^{\circ}$ respectively. If the precession angle for Fig. 2 were $3.5^{\circ}$, as stated, then one would expect from the reported cell constants of 94 and $80 \AA$ that respectively 7 and 6 orders of diffraction should appear in the inner circle of the pattern with $\mathrm{CuK} \alpha$ radiation. Instead, there are only 5 orders in each direction and the ratio of the spacings in this net is actually $1.09 \pm 0.01$, not 1.18 as for the reported parameters. Also, this diffraction pattern shows $m m$ symmetry, even in upper levels; thus the angle $\gamma$ must be $90^{\circ}$ exactly not approximately so as stated.

If the published account cannot be believed, then what is the true identity of the crystal that produced Fig. 2? In principle, the lattice parameters of a crystal can be determined from a well aligned screenless small-angle precession photograph such as this. Normally one would know the precession angle $\mu$ and the effective magnification factor $F$ (the product of crystal-to-film distance and photographic enlargement), but in this case these too are unknowns.

Fortunately, these parameters as well as the interplanar spacing $d$ that is normal to the aligned nets can be determined from the bounds of upper levels in the photograph. It can be shown that diffraction from the $n$th layer occurs on the film in an annulus bounded by limiting radii of

$$
\begin{gathered}
R_{n}\left(\begin{array}{c}
\text { outer } \\
\text { inner }
\end{array}\right)= \\
F\left\{\cos \mu \tan \left[\cos ^{-1}\left(\cos \mu-\frac{n \lambda}{d}\right)\right] \pm \sin \mu\right\}
\end{gathered}
$$

where $\lambda$ is the $\mathrm{X}$-ray wavelength. Lattice dimensions within the aligned nets can be obtained from the spacings $X_{n}$ in the $n$th annulus on the film according to

$$
a=\frac{F \lambda}{(1-n \lambda / d)} \cdot \frac{1}{\bar{X}_{n}}
$$

These formulae assume perfect alignment. However, misalignment error of the magnitude present in Fig. 2 of ref. 1 causes negligible perturbations. In this case, all unit cell angles must be $90^{\circ}$ since the pattern shows $\mathrm{mm}$ symmetry throughout.

Lorentz factor enhancement at the bounding edges of diffraction annuli in the precession geometry makes the limiting radii in a diffraction pattern readily measurable. These were at 8.1, 23.2, 31.4, $36.1,44.0$ and $46.5 \mathrm{~mm}$ respectively for $R_{0}^{\text {outer }}, R_{1}^{\text {inner }}, \ldots R_{3}^{\text {inner }}$ in a photocopy of Fig. 2 from the journal page. A leastsquares fit of equation 1 to these data gave $F=89 \pm 4 \mathrm{~mm}, d=35 \pm 3 \AA$ and $\mu=2.6$ $\pm 0.1^{\circ}$ with an r.m.s. residual of $0.12 \mathrm{~mm}$. When constrained to $\mu=2.5^{\circ}$, the nearest likely setting in common laboratory practice, the fit produced $F=92.1 \pm 1.2$ $\mathrm{mm}$ and $d=37.8 \pm 0.8 \AA$ with an r.m.s. residual of $0.14 \mathrm{~mm}$. Spacings in the first annulus were $X_{1}=1.986 \pm 0.007 \mathrm{~mm}$ vertically and $Y_{1}=1.882 \pm 0.006 \mathrm{~mm}$ horizontally. Then, by equation 2 , these values together with parameters from the $\mu$ $=2.5^{\circ}$ fit of equation 1 to the radii measurements gave lattice constants of $a=$ $74.4 \pm 1.0, b=81.4 \pm 1.1$ and $c=37.8 \pm$ $0.8 \AA$. As the cell is clearly primitive and orthorhombic and we assume that handed biological molecules were crystallized, the space group is necessarily either $P 2_{1} 2_{1} 2_{1}$, P222 or a permutation of $P 22_{1} 2$ or P222

When tentative crystallographic parameters had been determined we communicated them (without other comment) to Dr Gary Gilliland at the National Institutes of Health, whom we knew to be compiling a library of crystal data on macromolecules, and asked that he search for any crystal having these dimensions and symmetry. Only human carbonic anhydrase B (HCAB) of the nearly 700 entries in his data base at that time came close to having these dimensions. HCAB crystallizes in space group $P 2_{1} 2_{1} 2_{1}$ and its reported cell constants are $a=81.5, b=73.6$ and $c=$ $37.1 \AA$ (refs 2,3). At this point, Fig. 2 was checked against extant X-ray photographs of HCAB in Uppsala. Although such a screenless precession photograph could not be found, the relative diffraction intensities in (hk0), (hk $I$ ) and hk2) nets were seen to agree well with the published pattern. This leads us to the unambiguous conclusion that Fig. 2 of ref. 1 is an X-ray photograph of an HCAB crystal.

We have considered the possibility that the crystals were actually grown from a sample of carbonic anhydrase that had inadvertently been mistaken for one of tRNA. Paradies certainly had access to HCAB when he worked on TRNA in the Wallenberg Laboratory. However, such a mistake could not be the fault since the reported conditions for crystallization differ markedly from those needed for HCAB. HCAB crystals grow from a $2.3 \mathrm{M}$ ammonium sulphate solution at $p \mathrm{H} 8.7$ (ref.2) whereas the valyl RNA crystals are said to grow from 20 per cent solution of dioxane-water at $p \mathrm{H} 7.5$ (ref.1).

We also reject the hypothesis that HCAB photographs were accidentally inserted in the place of true tRNA patterns. It seems unacceptably implausible that such a mistake could occur and then remain undetected when Paradies proof-read and subsequently cited the article. Thus we are forced to conclude that the misrepresentation was intentional. The coauthor of the paper in question was not involved in this deception and did not see the manuscript before its publication. His role in the study was limited to supplying purified tRNA. $\mathrm{He}$ is not a crystallographer and had no reason to believe that Fig. 2 was wrong.

This is not an isolated incident. We have extensive documentation of similar misrepresentations in other articles published by Paradies. In one ${ }^{4}$, diffraction patterns from crystals of human carbonic anhydrase C (HCAC) are falsely represented as being from seryl tRNA crystals. In another ${ }^{5}$, a diffraction photograph from a crystal of HCAC is falsely described as one from a crystal of valyl-tRNA synthetase from Escherichia coli. Two other papers ascribe the same optical transform and inverted photographic images of the same electron micrograph to very different substances. In one case ${ }^{6}$ they are attributed to a negatively stained thin section from a crystal of algal D-ribulose 1,5-biphosphate carboxylase, and in the other ${ }^{7}$ a stained thin crystal of spinach choroplast coupling factor. One or both of these publications must be false, but there is no reason to suspect any complicity by coauthors of ref. 6 in any misrepresentation. Still other papers ${ }^{8,9}$ contain such serious flaws (for example major inconsistencies between descriptions in the text and the raw data in figures) that we believe they should discounted even though we cannot prove them fraudulent. WAYNE A. HENDRICKSON Laboratory for the Structure of Matter, Naval Research Laboratory, Washington DC, USA

Bror E. StrandberG ANDERS LILJAS

Wallenberg Laboratory,

University of Uppsala, Sweden

$$
\text { L. Mario AMZEL }
$$

EATON E. LATTMAN

Department of Biophysics,

Johns Hopkins University School of Medicine, Baltimore, Maryland, USA

\footnotetext{
1. Paradies, H.H. \& Sjöquisı, J. Nature 226, 159-161 (1970)

2. Kannan, K.K. et al. J. molec. Biol, 63, 601-604 (1972). Kannan, K.K. et al. Proc. natn. Acad. Sci. USA 72, 51-55 (1975)

4. Paradies, H.H. Eur. J. Biochem. 18, 530-535 (1971). 5. Paradies, H.H. J. Biochem., Tokyo 76, 655-659 (1974) 6. Zimmer, B., Paradies, H.H. \& Werz, G. Biochem. biophys. Res. Commun. 74, 1496-1500 (1977). Paradies, H.H. Biochem. biophys. Res. Commun. 91. 685-692 (1979).

8. Paradies, H.H. FEBS Lett. 2, 112-114 (1968)

Paradies, H.H. Biochem. biophys. Res. Commun. 92 $1076-1082(1980)$
}

A reply by Professor Paradies follows overleaf. 